Phytoplankton biomass and residual nitrate in the pelagic ecosystem

Platt, Trevor and Broomhead,

David S. and Sathyendranath, Shubha and Edwards, Andrew M. and Murphy, Eugene J.

2003

MIMS EPrint: 2006.19

Manchester Institute for Mathematical Sciences

School of Mathematics

The University of Manchester

\footnotetext{
Reports available from: http://eprints.maths.manchester.ac.uk/

And by contacting: The MIMS Secretary

School of Mathematics

The University of Manchester

Manchester, M13 9PL, UK
} 


\title{
Phytoplankton biomass and residual nitrate in the pelagic ecosystem
}

\author{
By Trevor Platt ${ }^{1}$, David S. BroomheAD ${ }^{2}$,
} Shubha Sathyendranath ${ }^{3,1}$, Andrew M. Edwards ${ }^{1,3}$

AND EUGENE J. MURPHY

${ }^{1}$ Biological Oceanography Section, Bedford Institute of Oceanography, Box 1006, Dartmouth, Nova Scotia B2Y 4A2, Canada

${ }^{2}$ Department of Mathematics, University of Manchester Institute of Science and Technology, PO Box 88, Manchester M60 1QD, UK

${ }^{3}$ Department of Oceanography, Dalhousie University, Halifax, Nova Scotia B3H 4J1, Canada

${ }^{4}$ British Antarctic Survey, Natural Environmental Research Council, Madingley Road, Cambridge CB3 OET, UK

Received 21 March 2002; revised 6 September 2002; accepted 8 October 2002; published online 12 February 2003

We develop and analyse a simple, two-compartment (chlorophyll and nitrate) model of the surface mixed layer of the ocean. The mixed-layer depth is modulated intermittently to simulate the effects of storms. The optical properties of the water column are linked to changes in the chlorophyll biomass. The model can be treated analytically. Mathematical bounds are found for the autotrophic biomass and the residual nitrate in terms of the intensity and frequency of storms and the bio-optical properties of the phytoplankton. The results are discussed in the context of the highnutrient, low-chlorophyll regimes, where unconsumed nitrate is a persistent occurrence.

Keywords: ocean modelling; high nutrient, low chlorophyll;

difference equations; coupled maps

\section{Introduction}

In the ocean, the chemical cycles of carbon and nitrogen are closely coupled. As nitrogen is, in general, a limiting resource for the growth of new plant material in the sea, any nitrate resupplied to the surface layer can be expected to be incorporated into autotrophic biomass (for which the operational index is concentration of chlorophyll). However, regimes do exist where elevated nitrate concentrations and depressed chlorophyll concentrations represent the quasi-permanent condition. These are the so-called HNLC (high-nutrient, low-chlorophyll) regimes (Chisholm \& Morel 1991).

In this context it is of interest to calculate the expected concentrations of chlorophyll and nitrate under different conditions of physical forcing. In particular, forcing by storms is important. Here, we develop and analyse a simple model of autotrophic 
(a)
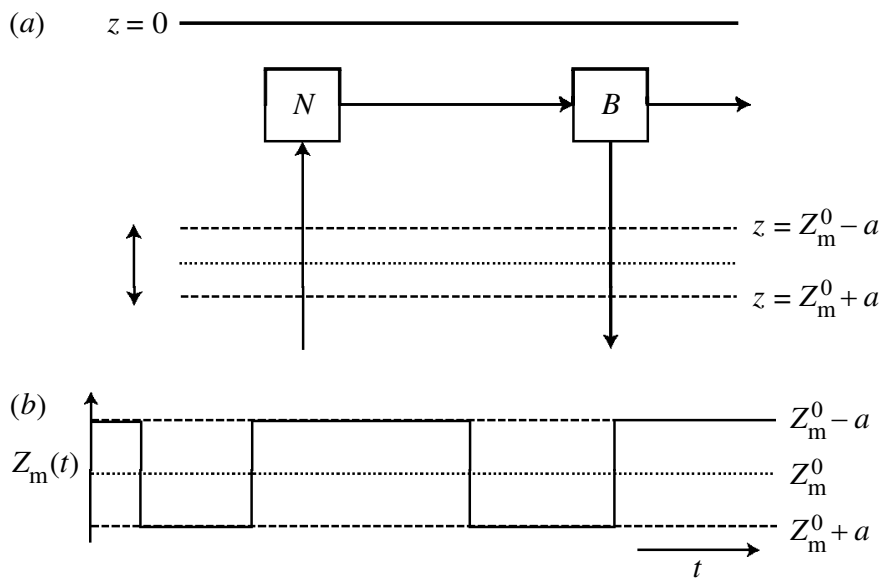

Figure 1. Schematic for the model discussed. (a) Nitrate ' $N$ ' is supplied from below the mixed layer and is converted to biomass ' $B$ ' of phytoplankton. (b) The mixed-layer depth $Z_{\mathrm{m}}(t)$ is modulated intermittently in a series of steps of common amplitude $2 a$, but variable time interval.

biomass and nitrate in the mixed layer of the ocean. Nitrate is resupplied intermittently through modulation of the mixed-layer depth, for example by the passage of storms. The analysis leads to mathematical bounds on the autotrophic biomass and residual nutrient concentration.

\section{A simple phytoplankton-nutrient model}

The model (figure 1a) is the simplest possible representation of dynamics in the pelagic ecosystem. Provided that there is sufficient nutrient (nitrate), growth of phytoplankton can occur in the mixed layer, possibly increasing the biomass (if growth exceeds the sum of losses) with a related decrease in the optical transparency. Below the mixed layer there is an infinite reservoir of nutrient at fixed concentration. Deepening of the mixed layer entrains nutrients into it, replacing, at least partly, the nutrients consumed by growth. Biomass of phytoplankton is assumed to be zero below the mixed layer, such that a deepening dilutes the biomass in the mixed layer with the entrained water, and therefore increases the transparency. Shallowing of the mixed layer has no effect on the concentrations of either phytoplankton or nutrients in the layer, but does result in a loss of the total quantities of each entity within the layer. Biomass lost by shallowing has no possibility of re-entering the mixed layer.

In the analysis, quantities are calculated on a daily basis. Let $B(t)$, measured in units of chlorophyll concentration, be the biomass of phytoplankton at the beginning of day $t$. Let $N_{\mathrm{d}}$ be the concentration of nutrients in the deep reservoir (independent of time), and let $N(t)$ be that in the mixed layer. Let $Z_{\mathrm{m}}(t)$ be the depth of the mixed layer; it will be modulated through simple step functions. Other quantities to be defined include the photosynthesis parameters $\alpha^{\mathrm{B}}$ (initial slope of the productionirradiance curve, where the superscript ' $\mathrm{B}$ ' indicates normalization to biomass) and $P_{\mathrm{m}}^{\mathrm{B}}$ (specific production at saturating light), the daylength $D$, and the surface irradiance at noon, $I_{0}^{\mathrm{m}}$ (Platt \& Sathyendranath 1993). The scaled noon irradiance $I_{*}^{\mathrm{m}}$ is $I_{*}^{\mathrm{m}}=I_{0}^{\mathrm{m}} \alpha^{\mathrm{B}} / P_{\mathrm{m}}^{\mathrm{B}}$. The diffuse vertical attenuation coefficient $K$ depends on the 
biomass according to $K(t)=K_{\mathrm{w}}+k_{\mathrm{c}} B(t)$, where $K_{\mathrm{w}}$ is the attenuation due to water and $k_{\mathrm{c}}$ is the specific absorption coefficient of chlorophyll.

Biomass $B$ increases through growth by photosynthesis and decreases by dilution and by a suite of loss terms. In the simplest case, these can be taken to be a fixed proportion $\lambda$ of the biomass. The daily (new) primary production of the mixed layer is $P(t)$, calculated according to the canonical form

$$
P(t)=P(B(t))=\frac{P_{\mathrm{m}}^{\mathrm{B}} B(t) D}{K(t)}\left[f\left(I_{*}^{\mathrm{m}}\right)-f\left(I_{*}^{\mathrm{m}} \mathrm{e}^{-K(t) Z_{\mathrm{m}}(t)}\right)\right],
$$

where $f(\cdot)$ is a known function of the normalized irradiance (Platt \& Sathyendranath 1993). The rate of primary production does not depend on nitrate concentration, except in so far as $P(t)$ will be zero whenever $N(t)$ is zero. The stoichiometric equivalents of nitrogen and carbon, relative to chlorophyll, are $\nu$ and $\chi$, respectively. The increment in biomass through photosynthesis in one day is then

$$
\frac{P(t)}{\chi Z_{\mathrm{m}}(t)}=\frac{P_{\mathrm{m}}^{\mathrm{B}} B(t) D}{\chi \theta(t)}\left[f\left(I_{*}^{\mathrm{m}}\right)-f\left(I_{*}^{\mathrm{m}} \mathrm{e}^{-\theta(t)}\right)\right],
$$

where $\theta(t)=Z_{\mathrm{m}}(t) K(t)$ is the optical thickness of the mixed layer.

The model is analysed through a coupled pair of iterative, non-autonomous maps, one for chlorophyll and one for nitrate,

$$
\begin{aligned}
& B(t+1)=\left[(1-\lambda) B(t)+\frac{P(t)}{\chi Z_{\mathrm{m}}(t)}\right](1-\Delta(t)), \\
& N(t+1)=N(t)-\left(\frac{\nu P(t)}{\chi Z_{\mathrm{m}}(t)}\right)(1-\Delta(t))+\left(N_{\mathrm{d}}-N(t)\right) \Delta(t),
\end{aligned}
$$

where modulation of the mixed-layer depth is represented by the quantity $\Delta(t)$, which takes non-zero values only when the mixed layer deepens:

$$
\Delta(t)= \begin{cases}\frac{Z_{\mathrm{m}}(t+1)-Z_{\mathrm{m}}(t)}{Z_{\mathrm{m}}(t+1)}, & \text { if } Z_{\mathrm{m}}(t)<Z_{\mathrm{m}}(t+1), \\ 0, & \text { if } Z_{\mathrm{m}}(t) \geqslant Z_{\mathrm{m}}(t+1) .\end{cases}
$$

The value of $Z_{\mathrm{m}}$ fluctuates about a value $Z_{\mathrm{m}}^{0}$ through a series of steps of common amplitude $2 a$, but with arbitrary width and alternating signs (figure $1 b$ ). These changes in mixed-layer depth, when they occur, are assumed to occur at the end of the day. We define an integer index $\xi$ to count the number of times the mixed layer deepens:

$$
\xi(t+1)= \begin{cases}\xi(t)+1, & \text { if } Z_{\mathrm{m}}(t)<Z_{\mathrm{m}}(t+1), \\ \xi(t), & \text { if } Z_{\mathrm{m}}(t) \geqslant Z_{\mathrm{m}}(t+1) .\end{cases}
$$

Then, the number of increases in the thickness of the mixed layer between time $t_{1}$ and a later time $t_{2}$ is just $\xi\left(t_{2}\right)-\xi\left(t_{1}\right)$. Successive deepening on successive time steps is not allowed, such that $\xi(t) \leqslant[(t+1) / 2] \leqslant(t+1) / 2$, where the square brackets indicate the integer part of the quantity enclosed.

Equations (2.3) and (2.4) may be added together to yield the composite variable $Q(t)=B(t)+N(t) / \nu$, which we may call the potential biomass, to be interpreted as 
the total of the standing biomass and the biomass equivalent of the residual nitrate. It evolves according to

$$
Q(t+1)=(1-\Delta(t)) Q(t)+\nu^{-1} N_{\mathrm{d}} \Delta(t)-\lambda B(t)(1-\Delta(t)) .
$$

In what follows, we first solve equation (2.7) for $Q(t)$ and deduce an explicit lower bound for the nitrate $N(t)$. Next, we examine the behaviour of $B(t)$ in the intervals between changes in mixed-layer depth: in particular, we solve the fixed-point equation for $B$ (in which the same value of $B(t)$ recurs for repeated iterations of the map). A stability analysis on the fixed points leads to an explicit upper bound for $B$.

\section{Solutions: bounds on biomass and residual nutrient}

For a given sequence of mixed-layer forcing $\{\Delta(t)\}$ and corresponding sequence of biomass $\{B(t)\}$, it can be shown by induction that the iterated map (2.7) has the solution

$$
Q(t)=Q(0) \prod_{s=0}^{t-1}(1-\Delta(s))+\sum_{s=0}^{t-1}\left[\nu^{-1} N_{\mathrm{d}} \Delta(s)-\lambda(1-\Delta(s)) B(s)\right] \prod_{s^{\prime}=s+1}^{t-1}\left(1-\Delta\left(s^{\prime}\right)\right) .
$$

A product $\prod$ is taken to be unity if the upper limit is smaller than the lower limit. Because

$$
\prod_{s=0}^{t-1}(1-\Delta(s))=G^{\xi(t)} \text { and } \quad \prod_{s^{\prime}=s+1}^{t-1}\left(1-\Delta\left(s^{\prime}\right)\right)=G^{\xi(t)-\xi(s+1)},
$$

where $G=\left(Z_{\mathrm{m}}^{0}-a\right) /\left(Z_{\mathrm{m}}^{0}+a\right)$, equation (3.1) simplifies to

$$
Q(t)=Q(0) G^{\xi(t)}+\sum_{s=0}^{t-1}\left[\nu^{-1} N_{\mathrm{d}} \Delta(s)-\lambda(1-\Delta(s)) B(s)\right] G^{\xi(t)-\xi(s+1)} .
$$

Furthermore,

$$
\sum_{s=0}^{t-1} \Delta(s) G^{\xi(t)-\xi(s+1)}=G^{\xi(t)} \sum_{s=0}^{t-1} \Delta(s) G^{-\xi(s+1)} .
$$

From the definitions of $(2.5)$ and (2.6) we see that $\Delta(s)$ is non-zero, such that $\Delta(s)=$ $2 a /\left(Z_{\mathrm{m}}^{0}+a\right)$, only when the mixed layer deepens between time-steps $s$ and $s+1$, for which $\xi(s+1)=\xi(s)+1$; note that $\Delta(s)$ refers to a deepening that is about to occur, whereas $\xi(s+1)$ refers to a deepening that has just occurred. The number of deepenings up to time $t$ is, by definition, $\xi(t)$, such that (using $j$ to index the deepenings)

$$
\begin{aligned}
G^{\xi(t)} \sum_{s=0}^{t-1} \Delta(s) G^{-\xi(s+1)} & =G^{\xi(t)} \frac{2 a}{Z_{\mathrm{m}}^{0}+a} \sum_{j=1}^{\xi(t)} G^{-j} \\
& =G^{\xi(t)} \frac{2 a}{Z_{\mathrm{m}}^{0}+a} \frac{G^{-\xi(t)}-1}{1-G}
\end{aligned}
$$


where we have used the general result that the sum of a geometric series is $\sum_{j=1}^{n} G^{-j}=\left(G^{-n}-1\right) /(1-G)$. Since $1-G=2 a /\left(Z_{\mathrm{m}}^{0}+a\right)$, we can cancel and then substitute

$$
\sum_{s=0}^{t-1} \Delta(s) G^{\xi(t)-\xi(s+1)}=1-G^{\xi(t)}
$$

into (3.2) to give

$$
Q(t)=Q(0) G^{\xi(t)}+\nu^{-1} N_{\mathrm{d}}\left(1-G^{\xi(t)}\right)-\lambda G^{\xi(t)} \sum_{s=0}^{t-1}(1-\Delta(s)) B(s) G^{-\xi(s+1)} .
$$

Suppose that there is a maximum interval $T$, say, between deepening events, where $T \geqslant 2$. Between time $t=0$ and $t \geqslant T$, there must be at least $[t / T]$ deepening events, and so $\xi(t) \geqslant[t / T]>(t / T)-1 ;[t / T]$ is the integer part of $t / T$. Then, noting that $0<G<1$, we see that $G^{\xi(t)}<G^{(t / T)-1}$, an upper bound for $G^{\xi(t)}$ which goes to zero for large $t$; then, to an arbitrarily good approximation,

$$
Q(t)=\nu^{-1} N_{\mathrm{d}}-\lambda G^{\xi(t)} \sum_{s=0}^{t-1}(1-\Delta(s)) B(s) G^{-\xi(s+1)} .
$$

We are now in a position to set bounds on the residual concentration of nitrate. Let us first assume that $B(t)$ is bounded above, $B(t) \leqslant B_{\mathrm{u}}$. Then, given that $Q(t)=$ $\nu^{-1} N(t)+B(t)$, we have

$$
\begin{aligned}
N(t)+\nu B(t) & =N_{\mathrm{d}}-\nu \lambda G^{\xi(t)} \sum_{s=0}^{t-1}(1-\Delta(s)) B(s) G^{-\xi(s+1)} \\
N(t) & =N_{\mathrm{d}}-\nu\left[B(t)+\lambda G^{\xi(t)} \sum_{s=0}^{t-1}(1-\Delta(s)) B(s) G^{-\xi(s+1)}\right] \\
& \geqslant N_{\mathrm{d}}-\nu B_{\mathrm{u}}\left[1+\lambda G^{\xi(t)} \sum_{s=0}^{t-1}(1-\Delta(s)) G^{-\xi(s+1)}\right] \\
& =N_{\mathrm{d}}-\nu B_{\mathrm{u}}\left[1+\lambda G^{\xi(t)} \sum_{s=0}^{t-1} G^{-\xi(s+1)}-\lambda G^{\xi(t)} \sum_{s=0}^{t-1} \Delta(s) G^{-\xi(s+1)}\right] \\
& =N_{\mathrm{d}}-\nu B_{\mathrm{u}}\left[1+\lambda G^{\xi(t)} \sum_{s=0}^{t-1} G^{-\xi(s+1)}-\lambda+\lambda G^{\xi(t)}\right] \\
& >N_{\mathrm{d}}-\nu B_{\mathrm{u}}\left[1+\lambda G^{\xi(t)} \sum_{s=0}^{t-1} G^{-\xi(s+1)}-\lambda\right] .
\end{aligned}
$$

From the observation that $\xi(t)-\xi(s+1)>(t-s-1-T) / T$, we obtain

$$
G^{\xi(t)-\xi(s+1)}<G^{(t-s-1-T) / T},
$$


such that

$$
\begin{aligned}
G^{\xi(t)} \sum_{s=0}^{t-1} G^{-\xi(s+1)} & <\sum_{s=0}^{t-1} G^{(t-s-1-T) / T} \\
& =G^{(t-T) / T} \sum_{s=0}^{t-1} G^{(-s-1) / T} \\
& =G^{t / T-1} \sum_{s=1}^{t} G^{-s / T} \\
& =G^{t / T-1}\left(G^{-t / T}-1\right) /\left(1-G^{1 / T}\right) \\
& =\left(G^{-1}-G^{t / T-1}\right) /\left(1-G^{1 / T}\right) \\
& <G^{-1} /\left(1-G^{1 / T}\right)
\end{aligned}
$$

where the last inequality will be almost an equality as $t$ gets large enough, such that $G^{t / T-1}$ is arbitrarily small. Then (3.9) becomes

$$
N(t)>N_{\mathrm{d}}-\nu B_{\mathrm{u}}\left[1+\lambda\left(\frac{1}{G\left(1-G^{1 / T}\right)}-1\right)\right] .
$$

The term multiplying $\lambda$ is strictly positive: therefore, the lower bound for the residual nitrate concentration will be higher for smaller maximum biomass and shorter intervals between storms.

Next, we consider conditions during the intervals between excursions of the mixed layer, and we find the equation for the fixed-point values $B_{*}$ of the biomass $B$. From equation $(2.3)$ it is

$$
B_{*}=(1-\lambda) B_{*}+\frac{P_{\mathrm{m}}^{\mathrm{B}} D B_{*}}{\chi \theta_{*}}\left[f\left(I_{*}^{\mathrm{m}}\right)-f\left(I_{*}^{\mathrm{m}} \mathrm{e}^{-\theta_{*}}\right)\right],
$$

where we have set $\theta_{*}=\left(K_{\mathrm{w}}+k_{\mathrm{c}} B_{*}\right) Z_{\mathrm{m}}$. Thus, there will be a fixed point at $B_{*}=0$. If we now cancel $B_{*}$ and rearrange equation (3.13) to put

$$
\theta_{*}=\frac{P_{\mathrm{m}}^{\mathrm{B}} D}{\chi \lambda}\left[f\left(I_{*}^{\mathrm{m}}\right)-f\left(I_{*}^{\mathrm{m}} \mathrm{e}^{-\theta_{*}}\right)\right],
$$

we see that there will be another (positive) fixed point, provided that the initial slope of the right-hand side of equation (3.14), with $\theta_{*}$ replaced by $\theta$, is greater than unity (figure $2 a$ ). This condition is equivalent to $\left(P_{\mathrm{m}}^{\mathrm{B}} D / \chi \lambda\right) I_{*}^{\mathrm{m}} f^{\prime}\left(I_{*}^{\mathrm{m}}\right)>1$, where the prime indicates differentiation. The fixed-point value is then

$$
B_{*}=\frac{\theta_{*}}{K_{\mathrm{c}} Z_{\mathrm{m}}}-\frac{K_{\mathrm{w}}}{K_{\mathrm{c}}} .
$$

For this fixed point to have physical meaning, we require that $B_{*}$ be positive, so that $\theta_{*}>K_{\mathrm{w}} Z_{\mathrm{m}}$. Equation (3.15) shows that the greater the mixed-layer depth, the smaller the steady-state biomass (figure $2 b$ ). Furthermore, from equation (3.14), it is clear that a (unique) solution can be found for $\theta_{*}$ in terms of the biological quantities $P_{\mathrm{m}}^{\mathrm{B}} D /(\chi \lambda)$ and $I_{*}^{\mathrm{m}}$ only. In particular, this solution will not depend on $Z_{\mathrm{m}}, k_{\mathrm{c}}$ or $K_{\mathrm{w}}$. 

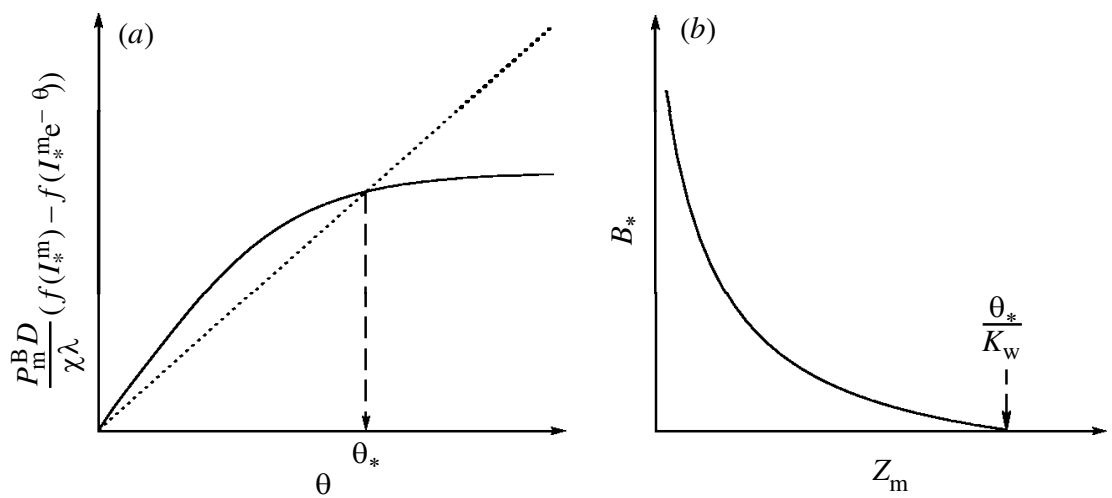

Figure 2. Solutions for the fixed points. (a) The right-hand side of equation (3.14) as a function of the optical thickness $\theta$ of the mixed layer. The dotted line is the one-to-one line $\theta=\theta$. The intersection of the two lines defines the positive fixed point $\theta_{*}$. (b) The fixed-point biomass $B_{*}$ as a function of $Z_{\mathrm{m}}$.

\section{Stability analysis}

Next, we examine the stability of the fixed points in the intervals between changes in mixed-layer depth, that is, during periods when $\Delta(t)=0$. This will be the case for most of the time when the coupled maps are iterated for many time steps. At such times, equation (2.3) can be rewritten as

$$
B(t+1)=B(t) \times F(B(t)),
$$

where

$$
F(B(t))=1-\lambda+\frac{P_{\mathrm{m}}^{\mathrm{B}} D}{\chi \theta(t)}\left[f\left(I_{*}^{\mathrm{m}}\right)-f\left(I_{*}^{\mathrm{m}} \mathrm{e}^{-\theta(t)}\right)\right] .
$$

The fixed points will now lie at $B_{*}=0$ and at some $B_{*}>0$ such that $F\left(B_{*}\right)=1$. The condition for the existence of the positive fixed point is $F(0)>1$, which is also the condition for the fixed point at $B_{*}=0$ to be unstable (see, for example, Drazin 1992; Edwards \& Bees 2001). For large $B$, it can be shown that $B F(B) \sim(1-\lambda) B+\left(P_{\mathrm{m}}^{\mathrm{B}} D / \chi k_{\mathrm{c}} Z_{\mathrm{m}}\right) f\left(I_{*}^{\mathrm{m}}\right)$, that is, a straight line with slope $(1-\lambda)<1$ (figure $3 a$ ). Therefore, the positive fixed point is stable and, moreover, because $B F(B)$ is increasing, the fixed point must be globally attracting: convergence to the fixed point will be non-oscillatory (Drazin 1992).

The resulting dynamics are as follows (figure $3 b$ ). From equation (3.15), we know that as $Z_{\mathrm{m}}$ increases, the value of $B_{*}$ at the positive fixed point decreases. Hence, the fixed-point value for $Z_{\mathrm{m}}=\left(Z_{\mathrm{m}}^{0}-a\right)$ will be greater than that when $Z_{\mathrm{m}}=\left(Z_{\mathrm{m}}^{0}+a\right)$. At a deepening event, $Z_{\mathrm{m}}(t)=\left(Z_{\mathrm{m}}^{0}-a\right)$ and then $Z_{\mathrm{m}}(t+1)=\left(Z_{\mathrm{m}}^{0}+a\right)$, with the term $\Delta(t) B(t)$ reducing the biomass by a factor $2 a /\left(Z_{\mathrm{m}}^{0}+a\right)$. The value of $B(t)$ then converges to the deep fixed point. On the other hand, when the layer shallows, there is no dilution of the biomass: the solution will start to converge to the shallow fixed point, but will not exceed it. This provides a value for the upper bound on the biomass,

$$
B_{\mathrm{u}}=\frac{\theta_{*}}{k_{\mathrm{c}}\left(Z_{\mathrm{m}}^{0}-a\right)}-\frac{K_{\mathrm{w}}}{k_{\mathrm{c}}}
$$




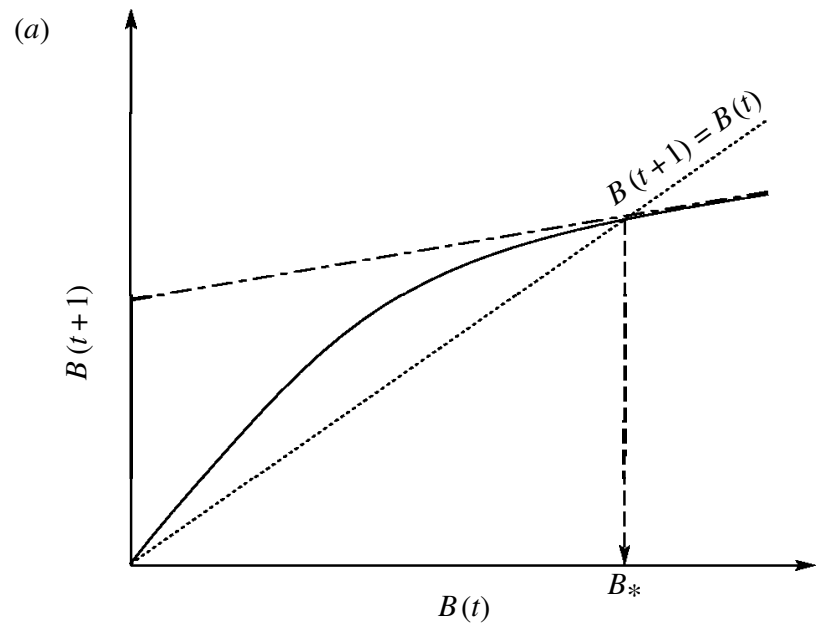

(b)

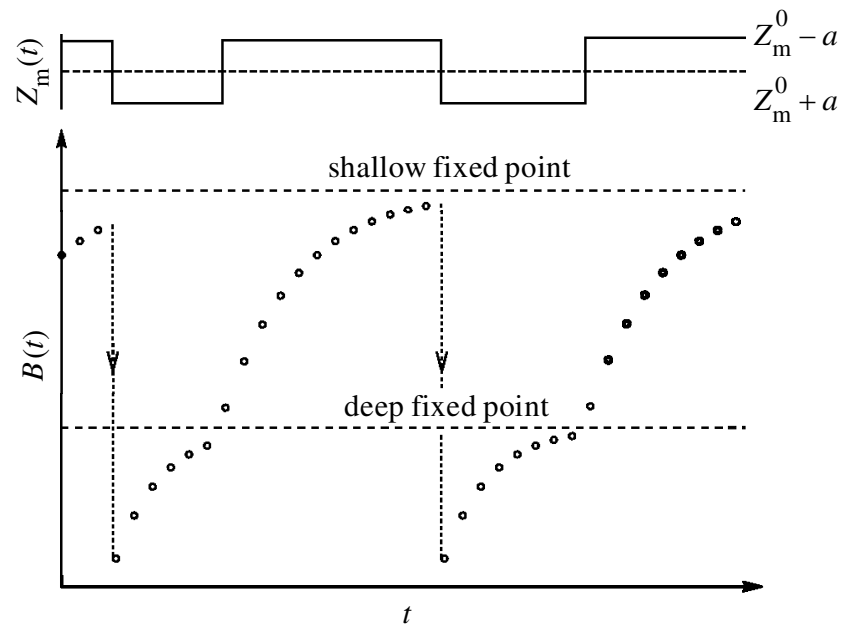

Figure 3. Stability analysis for the biomass. (a) Asymptotic solution for the biomass is a straight line with slope $(1-\lambda)<1$, showing that the positive fixed point is stable. (b) Convergence of the biomass to the fixed points corresponding to the two values of the mixed-layer depth. Deepening of the mixed layer dilutes the biomass to below its value at the deep fixed point, which it then approaches in a finite number of steps. Shallowing of the mixed layer causes the biomass to increase towards its value at the shallow fixed point.

Conversely, if the initial biomass exceeds the value of $B_{*}$ at the shallow fixed point, it will be reduced to this value within a finite number of iterations.

Another striking conclusion follows. Under conditions where the positive fixed point exists, consider the relative magnitudes of the growth term and the loss term, integrated over the mixed layer: they are exactly balanced when $Z_{\mathrm{m}}$ is the critical depth $Z_{\mathrm{m}}^{\mathrm{cr}}$ (in the sense of Sverdrup 1953). That is,

$$
\chi \lambda B(t) Z_{\mathrm{m}}^{\mathrm{cr}}(t)=P(t),
$$

where $P(t)$ is evaluated from equation (2.1) by substituting $Z_{\mathrm{m}}^{\mathrm{cr}}(t)$ for $Z_{\mathrm{m}}(t)$. We can now define a quantity $\theta^{\mathrm{cr}}(t)=\left(K_{\mathrm{w}}+k_{\mathrm{c}} B(t)\right) Z_{\mathrm{m}}^{\mathrm{cr}}(t)$. Given equations (2.1) and (4.4), $\theta^{\mathrm{cr}}(t)$ must satisfy a relation analogous to equation (3.14), for which there 
was a unique solution in terms of biological quantities. Therefore, we must have $\theta^{\operatorname{cr}}(t)=\theta_{*}$. Eliminating $\theta_{*}$ from equation (3.15) yields the simple result

$$
Z_{\mathrm{m}}^{\mathrm{cr}}(t)=Z_{\mathrm{m}}\left(\frac{K_{\mathrm{w}}+k_{\mathrm{c}} B_{*}}{K_{\mathrm{w}}+k_{\mathrm{c}} B(t)}\right) .
$$

The importance of this result is that, if the positive fixed point is attracting such that $B(t) \rightarrow B_{*}$ (and we have demonstrated that it is so), then the critical depth will be attracted to the mixed-layer depth.

\section{Interpretation}

We have presented a very simple model for the use of nitrate by phytoplankton in the upper mixed layer of the ocean. We have stressed the qualitative analysis of the equations rather than the simulations of many particular state-variable trajectories, partly to emphasize the generality of the solutions, but also in adherence to the view that analysis of progressively more complex cases is the key to understanding.

The simplifications that we have introduced are many, but we claim that our results would not be materially affected if these simplifications were removed at the expense of including more detail. Thus, we include no explicit grazing term. Grazing that is linear in the biomass would imply no more than a scale change in $\lambda$. Nonlinear grazing (see, for example, Fasham et al. 1990) would raise the possibility that the positive fixed point might become unstable, with the result that the sequence $B(t)$ could become oscillatory or even chaotic (Drazin 1992; Kuznetsov 1998). This is the opposite of what has been found for simple plankton ecosystem models that consist of differential equations, whereby changing the (zooplankton) loss term from a linear to a quadratic term tends to reduce the occurrence of oscillations (Edwards \& Yool 2000; Steele \& Henderson 1992). The critical depth would no longer be attracted to the mixed-layer depth if the (positive) fixed point were unstable. However, we have analysed the model with a quadratic loss term and used parameters corresponding to the Eastern Equatorial Pacific (Edwards et al. 2003); the fixed point does remain stable for all plausible values of the loss term.

Primary production is represented as independent of nitrate concentration unless nitrate concentration is zero, in which case primary production becomes zero. Inclusion of a Michaelis-Menten term to represent a reduction of primary production at low but positive nitrate concentrations would not affect the qualitative nature of the solutions. We have included no mechanism through which phytoplankton can grow on recycled nutrients. Thus, the analysis deals only with new production. By definition, regenerated production is the production required to sustain the metabolic demands of the entire pelagic community (Platt et al. 1989); in and of itself it cannot lead to an increment in biomass. However, the standing biomass utilizing reduced nitrogen will contribute to the optical density of the mixed layer, and it would therefore modify the quantitative solutions if not their qualitative nature.

For a given mixed-layer depth, our analysis shows that there will be a preferred value of biomass to which the simulated biomass will converge. Moreover, this preferred value is bounded, such that if the initial biomass is less than this bound, the biomass will approach but not exceed it. On the other hand, if the initial biomass is above the preferred value, it will be reduced to it within a finite number of steps. The magnitude of the preferred value for biomass is lower the greater the mixed-layer

Proc. R. Soc. Lond. A (2003) 
depth. Thus, the upper bound on mixed-layer biomass will coincide with the preferred value of the biomass at the minimum value of mixed-layer depth typical of the system. The solutions will go to the fixed points regardless of the initial conditions.

Corresponding to the upper bound on the biomass, there is an expected lower bound on the residual nutrient concentration. It will be higher for smaller upper bounds on the biomass (that is, deeper mixed layers), shorter intervals between storms, larger dynamic ranges for modulation of the mixed layer depth, and lower loss rates.

Regimes with residual (unconsumed) nitrate and relatively low phytoplankton biomass (that is HNLC regimes) may be expected whenever the local physical forcing so dictates. They need not be regarded as anomalies caused by the regional lack of some growth factor. Although there may be instances where evolution of the biological dynamics is constrained by the absence of some essential resource (such as iron), the unavailability of such a factor does not appear to be a necessary condition for the existence of HNLC regimes.

An unexpected result (albeit an understandable one with the benefit of hindsight), is that the Sverdrup critical depth is attracted to the mixed-layer depth. This means that, for given growth characteristics of the phytoplankton (and given loss rates), the biomass adjusts so that the Sverdrup criterion is just satisfied. If biomass would tend to increase beyond this point, the resultant change in the optical properties of the layer would decrease the critical depth relative to the mixed-layer depth, such that biomass would then tend to decrease. The converse would be true if biomass were reduced below the level that satisfies the Sverdrup condition. The important part played by the optical properties of the layer is clear.

That the critical depth is attracted to the mixed-layer depth raises the possibility, in particular applications, that the generalized loss term $L^{\mathrm{B}}$ could be quantified. Typically, the loss terms are much more difficult to assess than the growth terms (Platt et al. 1994). Assuming that the growth term were known, and that the critical depth could be taken to coincide with the mixed-layer depth, a solution for the loss terms would, in principle, be directly obtainable.

Recognition of the appearance of the Sverdrup critical depth (effectively, a statement of the conservation of mass on integrals over depth) in this analysis allows us to relate the results to those of earlier work. The Sverdrup criterion is a condition for the net growth of biomass in the mixed layer to be positive. It has been extended (Platt et al. 1991) to yield a time-scale for accumulation of bloom biomass, such that, despite the Sverdrup condition having been met, blooms will not occur unless this time is less than the typical interval between storms. We can now see that even though, according to some objective criterion (say, an increase in the biomass by a factor of ten), a bloom may occur, the maximum biomass attained could be constrained by the physical structure of the water column.

Now we should consider how the dynamics might respond if the physiology of the phytoplankton were perturbed, for example, by the addition of some putative limiting factor. We must distinguish (Banse 1990) between perturbation of the biomassnormalized rate $P / B$ and that of the absolute rate of production $P$. Observe that $P=B \times P / B$. Thus, an increase in the biomass-normalized rate, which would be expected as a first-order response to addition of a previously limiting growth factor, will not lead to an indefinite increase in biomass if the new fixed-point biomass is at some relatively low level and if it is prevented from straying away from this level

Proc. R. Soc. Lond. A (2003) 
by purely physical factors. It may be that the response would be to reach the fixedpoint biomass faster than in the unperturbed case. Depending on the taxa that would respond to the perturbation, another possible outcome is that the specific absorption coefficient $k_{\mathrm{c}}$ could change (Sathyendranath et al. 1999), leading to an increase in the upper bound $B_{\mathrm{u}}$ on the biomass through equation (4.3). These possibilities are explored in Platt et al. (2003), where we also show how the model applies to different oceanic regions.

The results presented here provide a common basis for the understanding of apparently disparate dynamics, and this work has shown the value of the analytic approach to the study of ocean biogeochemistry.

This work was initiated while the authors were in residence at the Isaac Newton Institute for the Mathematical Sciences, Cambridge. A.M.E. was supported by an NSERC Visiting Fellowship in a Canadian Government Laboratory and by the Department of Fisheries and Oceans Strategic Science Fund, project number 21778.

\section{References}

Banse, K. 1990 Does iron really limit phytoplankton production in the offshore subarctic Pacific? Limnol. Oceanogr. 35, 772-775.

Chisholm, S. W. \& Morel, F. M. M. (eds) 1991 What controls phytoplankton production in nutrient-rich areas of the open sea? Limnol. Oceanogr. 46, 1507-1970.

Drazin, P. G. 1992 Nonlinear systems. Cambridge University Press.

Edwards, A. M. \& Bees, M. A. 2001 Generic dynamics of a simple plankton population model with a non-integer exponent of closure. Chaos Solitons Fractals 12, 289-300.

Edwards, A. M. \& Yool, A. 2000 The role of higher predation in plankton population models. J. Plankton Res. 22, 1085-1112.

Edwards, A. M., Platt, T. \& Sathyendranath, S. 2003 A simple model of oceanic high-nutrient, low-chlorophyll regions to determine effects of iron fertilisation. (Submitted.)

Fasham, M. J. R., Ducklow, H. W. \& McKelvie, S. M. 1990 A nitrogen-based model of plankton dynamics in the oceanic mixed layer. J. Mar. Res. 48, 591-639.

Kuznetsov, Y. A. 1998 Elements of applied bifurcation theory, 2nd edn, vol. 112. Applied Mathematical Sciences. Springer.

Platt, T. \& Sathyendranath, S. 1993 Estimators of primary production for interpretation of remotely sensed data on ocean color. J. Geophys. Res. 98, 14561-14576.

Platt, T., Harrison, W. G., Lewis, M. R., Li, W. K. W., Sathyendranath, S., Smith, R. E. \& Vézina, A. F. 1989 Biological production of the oceans: the case for a consensus. Mar. Ecol. Prog. Ser. 52, 77-88.

Platt, T., Bird, D. F. \& Sathyendranath, S. 1991 Critical depth and marine primary production. Proc. R. Soc. Lond. B 246, 205-217.

Platt, T., Woods, J. D., Sathyendranath, S. \& Barkmann, W. 1994 Net primary production and stratification of the ocean. Geophys. Monogr. 85, 247-254.

Platt, T., Sathyendranath, S., Edwards, A. M., Broomhead, D. S. \& Ulloa, O. 2003 Nitrate supply and demand in the mixed layer of the ocean. Mar. Ecol. Prog. Ser. (In the press.)

Sathyendranath, S., Stuart, V., Irwin, B. D., Maass, H., Savidge, G., Gilpin, L. \& Platt, T. 1999 Seasonal variations in bio-optical properties of phytoplankton in the Arabian Sea. Deep-Sea Res. II 46, 633-654.

Steele, J. H. \& Henderson, E. W. 1992 The role of predation in plankton models. J. Plankton Res. 14, 157-172.

Sverdrup, H. U. 1953 On conditions for the vernal blooming of phytoplankton. J. Conseil 18, $287-295$. 